\title{
Supporting Information to: Magnesium impurities decide the structure of calcium carbonate hemihydrate
}

\author{
Julie Aufort, Raffaella Demichelis
}

Curtin Institute for Computation, The Institute for Geoscience Research (TIGER) and School of Molecular and Life Science, Curtin University, PO Box U1987, Perth, Western Australia,

Australia

Table S1. Cell parameters averaged over all combinations as a function of the Mg substitution content $x$ and standard deviation obtained from geometry optimisation at the classical level of theory (force field). A monoclinic cell was imposed for 3.125 and $6.25 \% \mathrm{Mg}$ substitutions.

\begin{tabular}{c|ccccccc}
\hline & $x=0$ & $x=0.03125$ & $x=0.0625$ & $x=$ & $x=0.9375$ & $x=0.96875$ & $x=1$ \\
& & $(\mathrm{SD})$ & $(\mathrm{SD})$ & 0.5 & $(\mathrm{SD})$ & $(\mathrm{SD})$ & \\
\hline $\mathrm{a}(\AA)$ & 9.384 & 9.382 & 9.378 & 9.144 & 9.051 & 9.0117 & 8.859 \\
& & $(0.003)$ & $(0.006)$ & & $(0.101)$ & $(0.089)$ & \\
$\mathrm{b}(\AA)$ & 10.505 & 10.472 & 10.436 & 9.945 & 9.385 & 9.351 & 9.307 \\
& & $(0.001)$ & $(0.010)$ & & $(0.031)$ & $(0.021)$ & \\
$\mathrm{c}(\AA)$ & 5.898 & 5.896 & 5.894 & 5.756 & 5.682 & 5.677 & 5.720 \\
& & $(0.003)$ & $(0.010)$ & & $(0.088)$ & $(0.013)$ & \\
$\alpha\left(^{\circ}\right)$ & 90.00 & 90.00 & 90.00 & 90.000 & 90.00 & 90.00 & 90.000 \\
$\beta\left(^{\circ}\right)$ & 90.00 & 90.093 & 90.117 & 91.818 & 90.196 & 90.188 & 90.000 \\
& & $(0.012)$ & $(0.085)$ & & $(0.138)$ & $(0.114)$ & \\
$\gamma\left({ }^{\circ}\right)$ & 90.00 & 90.00 & 90.00 & 90.000 & 90.00 & 90.00 & 90.000 \\
\hline
\end{tabular}




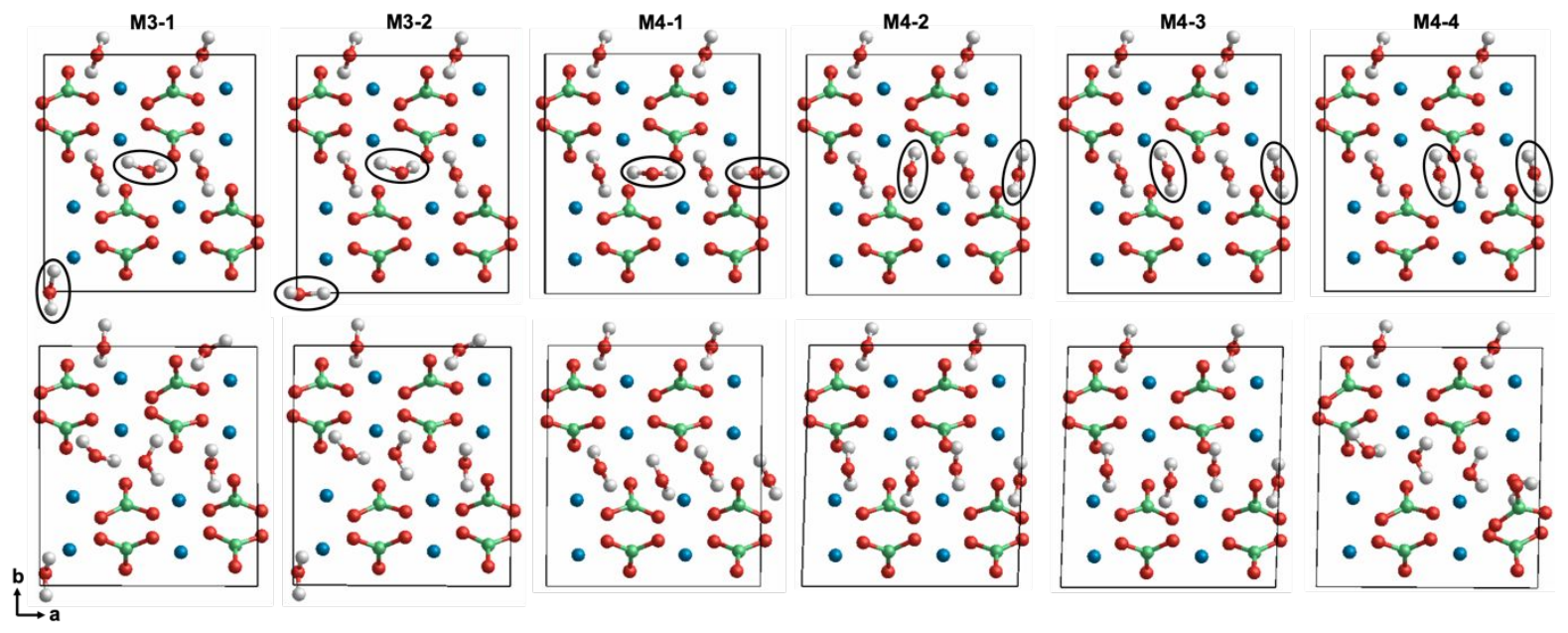

Figure S1. Initial configurations (top) and FF-optimised structures (bottom) for $\mathrm{CaCO}_{3} \cdot 0.75$ $\mathrm{H}_{2} \mathrm{O}$ starting from the experimental $P 2_{1} / c$ structure of calcium carbonate hemihydrate and viewed along [001]. The two additional water molecules are circled in black. Oxygen atoms are in red, calcium in blue, carbon in green and hydrogen atoms in white.
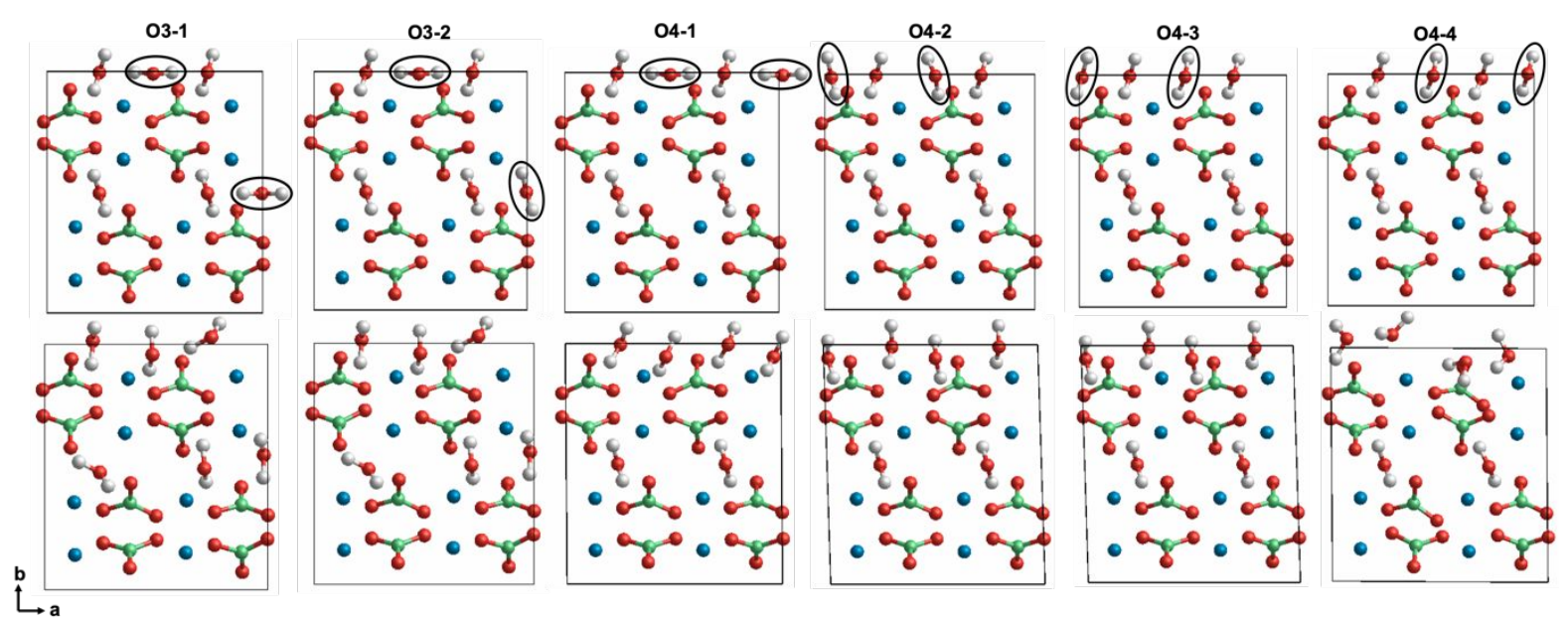

Figure S2. Initial configurations (top) and FF-optimised structures (bottom) for $\mathrm{CaCO}_{3} \cdot 0.75$ $\mathrm{H}_{2} \mathrm{O}$ starting from the optimised $\mathrm{Pbcn}$ structure of calcium carbonate hemihydrate and viewed along [001]. The two additional water molecules are circled in black. Colours as in figure S1. 


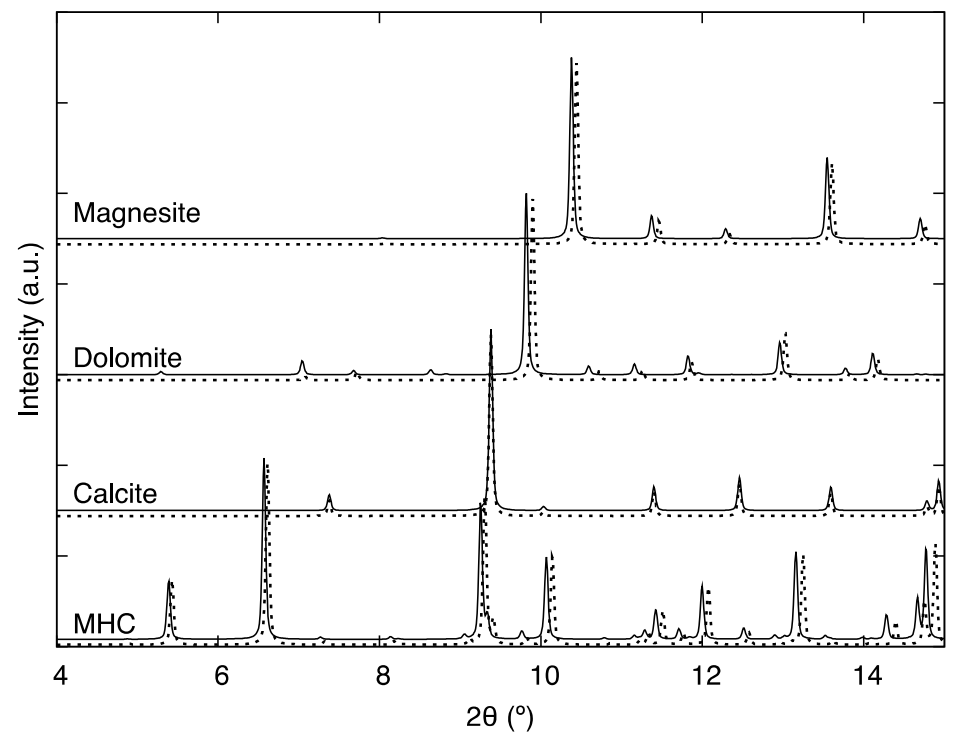

Figure S3. Simulated XRD patterns of magnesite, dolomite, calcite and monohydrocalcite obtained from experimental (solid lines) and DFT PBE0-DC optimised (dotted lines) structures. Patterns are shifted vertically for comparison.
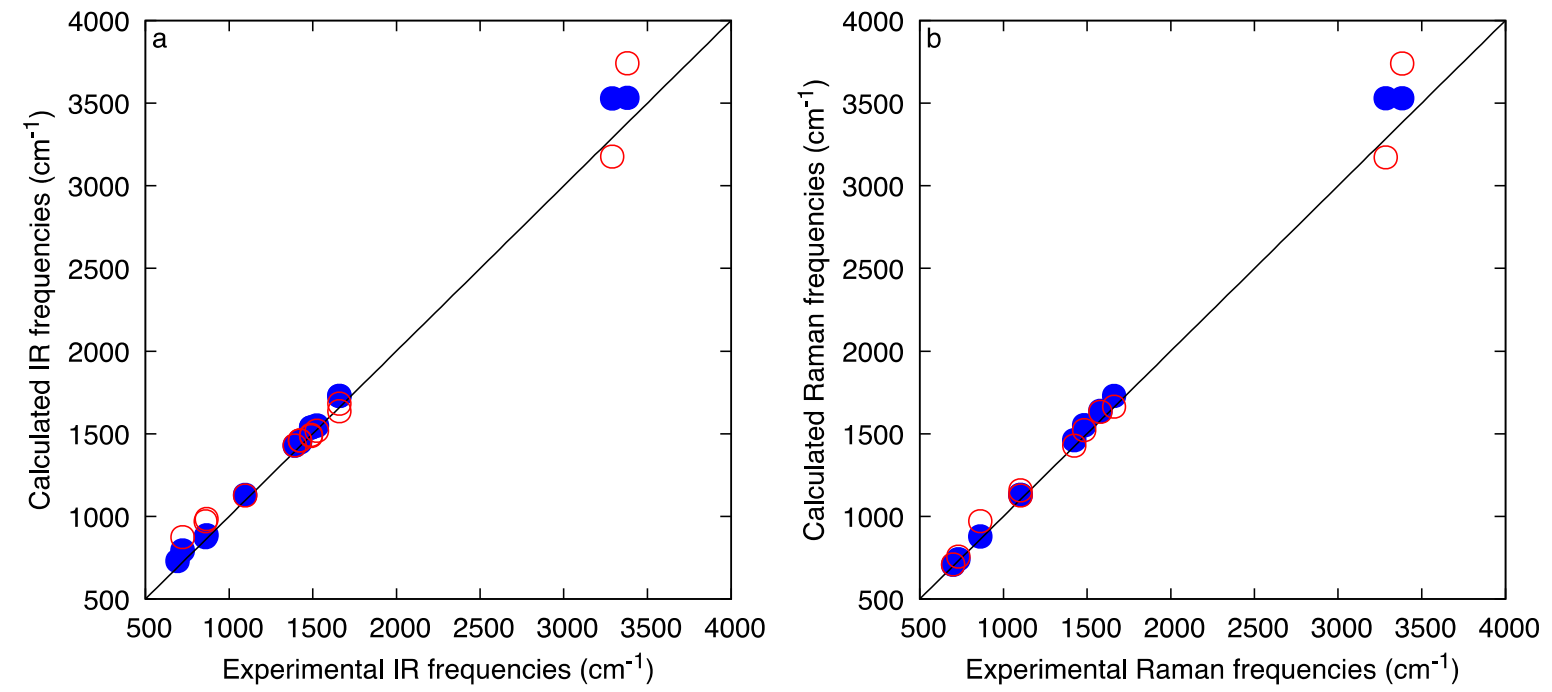

Figure S4 a. Experimental and calculated harmonic infrared frequencies of calcium (blue solid circles) and 50\% Mg-substituted (red open circles) carbonate hemihydrate. b. Experimental and calculated raman shifts of calcium (blue solid circles) and 50\% Mg-substituted (red open circles) carbonate hemihydrate. 

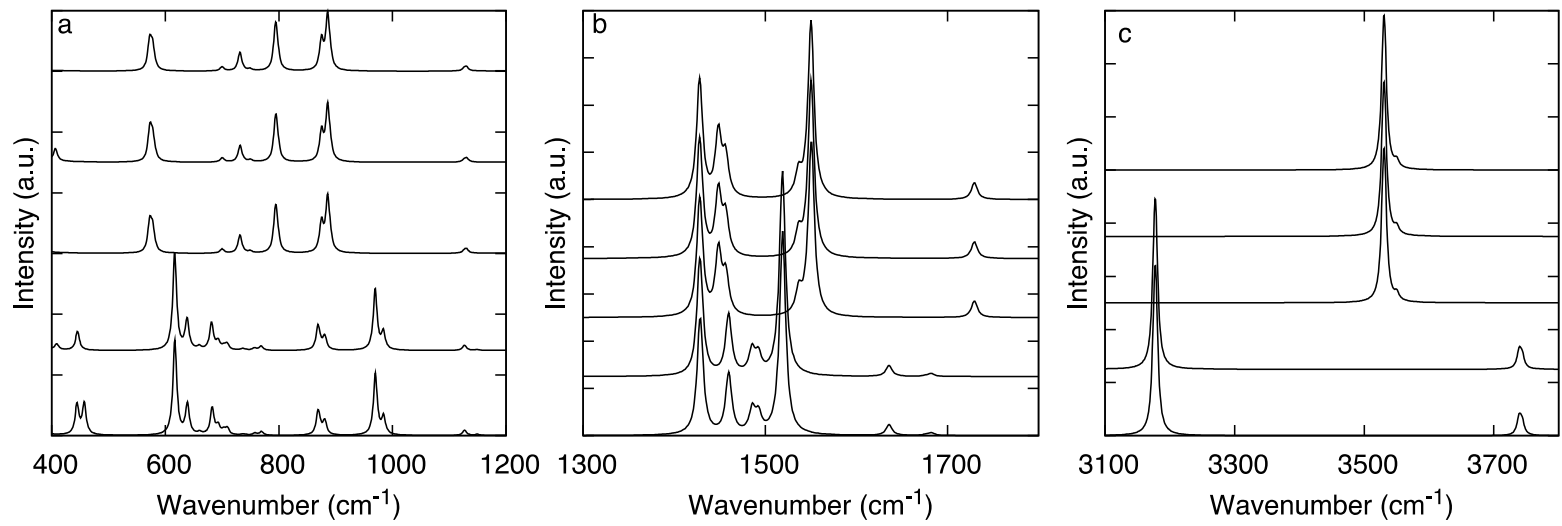

Figure S5. IR spectra of pure calcium carbonate hemihydrate and after isotopic substitution of $12.5 \%$ and $50 \% \mathrm{Ca}$ with the atomic mass of magnesium, and spectra of $50 \% \mathrm{Mg}$ substituted carbonate hemihydrate after isotopic substitution of all $\mathrm{Mg}$ atoms to $\mathrm{Ca}$ atomic mass, showing the structural $\mathrm{CO}_{3}$ bands in the $400-1200 \mathrm{~cm}^{-1}$ (a) and $1300-1800 \mathrm{~cm}^{-1}$ (b) ranges and $\mathrm{OH}$ stretching bands (c). Spectra are shifted vertically for comparison. 